\title{
Effects of Escherichia coli heat-stable enterotoxin and guanylin on the barrier integrity of intestinal epithelial T84 cells
}

\section{Ryo Nakashima, Yoichi Kamata and Yoshikazu Nishikawa}

\begin{tabular}{|c|l|}
\hline Citation & Veterinary immunology and immunopathology; 152(1-2): 78-81 \\
\hline Issue Date & $2013-3-15$ \\
\hline Type & Journal Article \\
\hline Textversion & author \\
\hline \multirow{3}{*}{ Right } & $\begin{array}{l}\text { C } 2012 \text { Elsevier B.V. This manuscript version is made available under the } \\
\text { CC-BY-NC-ND 4.0 License. http://creativecommons.org/licenses/by־nc-nd/4.0/ } \\
\text { This is the accepted manuscript version. The following manuscript has been accepted } \\
\text { by Veterinary immunology and immunopathology. The final, published version is } \\
\text { available at https://doi.org/10.1016/j.vetimm.2012.09.026. }\end{array}$ \\
\hline DOI & \begin{tabular}{l}
$10.1016 / j . v e t i m m .2012 .09 .026$ \\
\hline
\end{tabular}
\end{tabular}

Self-Archiving by Author(s)

Placed on: Osaka City University Repository

NAKASHIMA R, KAMATA Y, \& NISHIKAWA Y. (2013). Effects of Escherichia coli heat-stable enterotoxin and guanylin on the barrier integrity of intestinal epithelial T84 cells. Veterinary Immunology and Immunopathology. 152, 78-81. Doi: 10.1016/j.vetimm.2012.09.026 
Effects of Escherichia coli heat-stable enterotoxin and guanylin on the barrier integrity of intestinal epithelial T84 cells

Ryo Nakashima ${ }^{\mathrm{a}}$, Yoichi Kamata ${ }^{\mathrm{b}}$, and Yoshikazu Nishikawa ${ }^{\mathrm{a}}$

${ }^{a}$ Graduate School of Human Life Science, Osaka City University, Sugimoto 3-3-138, Osaka 558-8585, Japan

${ }^{\mathrm{b}}$ Division of Microbiology, National Institute of Health Sciences, Kamiyoga 1-18-1, Tokyo 158-8501, Japan

*Corresponding author: Yoshikazu Nishikawa

Department of Food and Human Health Sciences

Osaka City University Graduate School of Human Life Science

3-3-138 Sugimoto, Sumiyoshi-ku, Osaka 558-8585, Japan

Tel. \& Fax: +81 6-6605-2910

E-mail: nisikawa@life.osaka-cu.ac.jp 


\begin{abstract}
Tight junctions contribute to the formation and establishment of intestinal epithelial barriers against microbial infections. However, a variety of enteric pathogens have developed strategies to adhere to and invade epithelial cells and disrupt epithelial integrity. The aim of this study was to ascertain if enterotoxigenic $E$. coli heat-stable enterotoxin (STa) can cause deterioration of epithelial barrier integrity. Since STa shows amino acid similarity to guanylin, we evaluated the effects of both of these molecules on T84 epithelial cells. T84 epithelial monolayers were grown on 24-well Transwell filters and barrier integrity was assayed by measurement of transepithelial electrical resistance (TER). Macromolecular permeability of the monolayers was determined by measuring the paracellular passage of FITC-labeled dextran 4000 from apical to basolateral compartments of the Transwell filter culture. Treatment of T84 monolayers with either ST or guanylin did not increase paracellular permeability to FITC-dextran. However, although guanylin, which is a physiological guanylate cyclase activator, did not change TER in polarized T84 monolayers, ST did elicit a reduction in TER within $2 \mathrm{~h}$, at concentrations above $4 \mu \mathrm{M}$. These data suggest that STa causes not only induction of water secretion but also intestinal barrier dysfunction.
\end{abstract}

Keywords: enterotoxin; intestinal barrier; tight junction

\begin{abstract}
Abbreviations list
diarrheagenic E. coli (DEC), Dulbecco's Modified Eagle's Medium (DMEM), enterotoxigenic E. coli (ETEC), heat-stable enterotoxin (STa), tight junction (TJ), transepithelial electrical resistance (TER) heat-labile enterotoxin (LT), ethylene glycol tetra acetic acid (EGTA)
\end{abstract}




\section{Introduction}

Escherichia coli $($ E. coli) is the predominant facultative anaerobe of human colonic flora. Generally, most of the E. coli strains in the intestinal lumen of healthy people are harmless. However, some E. coli clones are virulent and can cause a spectrum of diseases such as sepsis, urinary tract infection, and diarrheal disease. Enterotoxigenic E. coli (ETEC) are some of the best-known diarrheagenic E. coli in both livestock and humans (Croxen and Finlay, 2010; Turner et al., 2006). These organisms colonize intestinal mucosa using species-specific colonization factors. However, heat-labile enterotoxin (LT) and heat-stable enterotoxin (STa) are present in both animal and human ETEC strains. LT and STa cause an increase in intraepithelial cAMP and cGMP levels, respectively, and subsequently mediate decreased absorption of sodium and chloride ions, and increased secretion of bicarbonate and chloride ions, via activation of the cystic fibrosis transmembrane conductance regulator (Fasano, 1999). Thus, ETEC cause watery diarrhea by changing epithelial function from absorption to secretion.

Tight junctions (TJ) of intestinal epithelial cells contribute to the formation and establishment of barriers between the intestinal lumen and subepithelial tissues. However, a variety of enteric pathogens have developed strategies to disrupt epithelial integrity as well as to adhere to and invade the epithelial cells (Bonazzi and Cossart, 2011). For example, Clostridium perfringens and $C$. difficile use toxins to induce the deterioration of tight junctions.

A reduction in transepithelial electric resistance and the paracellular passage of FITC-labeled dextran are recognized as indices of the decreased barrier integrity of epithelial cells infected with microbes. The objectives of this study were to assess whether these indices of barrier integrity could be reduced by STa and to compare the effects of STa on these indices with the effects of guanylin. A comparison with guanylin was carried out because the amino acid sequence 
of STa is functionally similar to that of the physiological modulator guanylin, which is known to activate guanylate-cyclase-C in intestinal epithelial cells and to induce water secretion from epithelia (Taxt et al., 2010).

\section{Materials and methods}

\subsection{Epithelial cell lines}

The T84 human colon cell line was routinely maintained in medium containing a 1:1 mixture of Dulbecco’s Modified Eagle’s Medium (DMEM) (Nissui Pharmaceutical, Tokyo, Japan) and Ham's F-12 (Nissui Pharmaceutical) supplemented with 2 mM L-glutamine, $0.15 \% \mathrm{NaHCO}_{3}, 5 \%$ FBS (Invitrogen, Grand Island, NY), 63 mg/ml penicillin (Sigma, St. Louis, MO), and 100 mg/ml streptomycin (Sigma). The cells were seeded at high density in $25 \mathrm{~cm}^{2}$ polystyrene tissue culture flasks and were maintained in humidified incubator with $5 \% \mathrm{CO}_{2}$ at $37^{\circ} \mathrm{C}$. To generate polarized T84 cell monolayers, approximately $4 \times 10^{4}$ cells were seeded onto $0.33 \mathrm{~cm}^{2}$ polystyrene Transwell permeable support cell culture inserts $(0.4 \mu \mathrm{m}$ pore size; Costar $)$ and were grown for 7-9 days. Transepithelial electrical resistance (TER) was calculated as $\Omega \cdot \mathrm{cm}^{2}$ using the electrical resistance measured with a Millicell Electrical Resistance System (Millipore, Bedford, MA) and the surface area of the filter. Monolayers were used for experiments in which TER was increased up to $>1,000 \Omega \cdot \mathrm{cm}^{2}$.

\subsection{Test for epithelial barrier integrity}


T84 cells were seeded on 24-well Transwell polyester cell culture inserts as described above. The growth medium in the apical and basolateral compartments of the Transwell inserts were exchanged for DMEM/F12 (1:1) containing 0.5\% FBS. After the medium change, T84 cells were maintained in $5 \% \mathrm{CO}_{2}$ at $37{ }^{\circ} \mathrm{C}$ for $2 \mathrm{~h}$ for equilibration. Peptides or chemicals were then added into the apical compartment of the Transwell inserts and TER was measured. The background reading of a cell-free control filter was subtracted from sample readings. Ethylene glycol tetra acetic acid (EGTA; Kanto Kagaku, Tokyo, Japan) treatment (4 mM) functioned as a positive control for induction of tight junction dilation and forskolin (Wako, Osaka, Japan) was used as a substitute for LT and a positive control for adenylate cyclase activation.

Paracellular macromolecular permeability through T84 cell monolayers was determined by measuring the paracellular passage of FITC-dextran (molecular weight $4 \mathrm{kDa}$ ) (Sigma) from apical to basolateral compartments of the Transwell filter culture. The test substance and the FITC-dextran $(2 \mathrm{mg} / \mathrm{ml})$ were dissolved in phenol red-free DMEM/F12 (1:1) containing 0.5\% FBS and the mixture $(150 \mu \mathrm{l})$ was loaded onto the apical side of the monolayer. The FITC-dextran concentration in the basolateral compartment was determined longitudinally on the basis of fluorescence intensity analyzed using a spectrofluorophotometer (Wallac 1420 ARVOSX; PerkinElmer Life Sciences, Boston, MA) at an excitation wavelength of $485 \mathrm{~nm}$ and an emission wavelength of $535 \mathrm{~nm}$.

\subsection{Assay of cGMP}

The cGMP level of T84 cells was measured using the Cyclic GMP EIA Kit (Cayman Chemical C., Ann Arbor, MI) after reaction of the cells with the test substance for $4 \mathrm{~h}$. 


\section{Results and Discussion}

T84 epithelial monolayers were treated with enterotoxin STa, and its effect on TER was compared with the effects of the endogenous peptide guanylin. Forskolin, a well-known cAMP inducer, was used as a positive control for decrease in TER (Fig. 1). STa reduced TER within $2 \mathrm{~h}$ at doses ranging between $4 \times 10^{-6}$ and $10^{-5} \mathrm{M}$, and this reduction was similar to that of forskolin. In contrast, the guanylin, an endogenous guanylate-cyclase-C ligand, showed no effect on TER. Neither STa nor guanylin increased paracellular permeability as assessed by measurement of the paracellular passage of FITC-dextran although, the FITC-dextran did pass through the epithelia in $8 \mathrm{~h}$ when EGTA was added as a positive control of TJ dilation (Fig. 2 A and B). Not only STa but also guanylin increased cellular cGMP within $4 \mathrm{~h}$ at doses between $10^{-6}$ and $10^{-5} \mathrm{M}$ (data not shown).

T84 epithelial monolayers were reconfirmed as a useful in vitro model for the detection of

effectors of TJ. Several studies have shown that toxins often have the ability to dilate TJ and increase paracellular permeability (Guttman and Finlay, 2009; Hofman, 2003; Soong et al., 2008). Both STa and forskolin (Fig. 2C) decreased TER but did not allow the passage of dextran-FITC, suggesting that both of these substances induced a moderate increase in paracellular permeability. In contrast, the physiological substance guanylin increased cGMP to the same level as that induced by STa, but did so without affecting TER. The observed effect of STa on TER is likely to play a role in the pathogenesis of ETEC.

It was recently reported that LT could cause not only a reduction in TER but also in the passage of dextran-FITC by disrupting the TJ of T84 cells (Kreisberg et al., 2011); the lack of 
dextran passage in the forskolin-treated cells would suggest that LT causes barrier permeability for large molecules in an cAMP independent way. On the other hand, STa-producing ETEC organisms did not affect the TER in that study. Since we observed a decrease in TER only when

STa was added to T84 cells at doses of more than $4 \times 10^{-6} \mathrm{M}$, it is possible that the STa-induced reduction of TER may only occur at the limited number of epithelial sites to which the ETEC organisms adhere and at which they elaborate the STa.

\section{Conclusion}

Although both STa and guanylin induced cellular cGMP production in T84 epithelial monolayers, only STa reduced barrier integrity. The effect of STa on epithelial tight junctions may contribute to enterotoxic activity.

\section{Acknowledgements}

A part of this study was financially supported by a grant from the Ministry of Health, Labour and Welfare of Japan.

\section{Conflict of Interest statement}

We declare that we have no conflict of interest in connection with this paper. 


\section{References}

Bonazzi, M., Cossart, P., 2011, Impenetrable barriers or entry portals? The role of cell-cell adhesion during infection. J. Cell Biol. 195, 348-357.

Croxen, M.A., Finlay, B.B., 2010, Molecular mechanisms of Escherichia coli pathogenicity. Nat. Rev. Microbiol. 8, 26-38.

Fasano, A., 1999, Cellular microbiology: can we learn cell physiology from microorganisms? Am. J. Physiol. 276, C765-776.

Guttman, J.A., Finlay, B.B., 2009, Tight junctions as targets of infectious agents. Biochim. Biophys. Acta. 1788, 832-841.

Hofman, P., 2003, Pathological interactions of bacteria and toxins with the gastrointestinal epithelial tight junctions and/or the zonula adherens: An update. Cell. Mol. Biol. 49, 65-75.

Kreisberg, R.B., Harper, J., Strauman, M.C., Marohn, M., Clements, J.D., Nataro, J.P., 2011, Induction of increased permeability of polarized enterocyte monolayers by enterotoxigenic Escherichia coli heat-labile enterotoxin. Am. J. Trop. Med. Hyg. 84, 451-455.

Soong, G., Parker, D., Magargee, M., Prince, A.S., 2008, The type III toxins of Pseudomonas aeruginosa disrupt epithelial barrier function. J. Bacteriol. 190, 2814-2821.

Taxt, A., Aasland, R., Sommerfelt, H., Nataro, J., Puntervoll, P., 2010, Heat-stable enterotoxin of enterotoxigenic Escherichia coli as a vaccine target. Infect. Immun. 78, 1824-1831.

Turner, S.M., Scott-Tucker, A., Cooper, L.M., Henderson, I.R., 2006, Weapons of mass destruction: virulence factors of the global killer enterotoxigenic Escherichia coli. FEMS Microbiol Lett 263, 10-20. 


\section{Figure legends}

Fig. 1. Effect of forskolin, ST and guanylin on longitudinal changes in transepithelial electrical resistance (TER) through T84 monolayers. Forskolin (A), STa (B), or guanylin (C) were added to T84 monolayers and incubated for the indicated times. TER was then measured using a Millicell Electrical Resistance System (Millipore). Forskolin, an adenylate cyclase activator, was used as a substitute for the heat-labile enterotoxin of ETEC. EGTA, which loosens tight junctions, was used as a positive control.

Fig. 2. Effects of ST and guanylin on macromolecular paracellular permeability of T84 monolayers. Cell permeability was longitudinally determined by measuring the paracellular passage of FITC-labelled dextran 4000 from apical to basolateral compartments of the Transwell filter culture after addition of STa (A), guanylin (B) or forskolin (C) to the apical side. EGTA, which loosens tight junctions, was used as a positive control. 


\section{Forskolin}

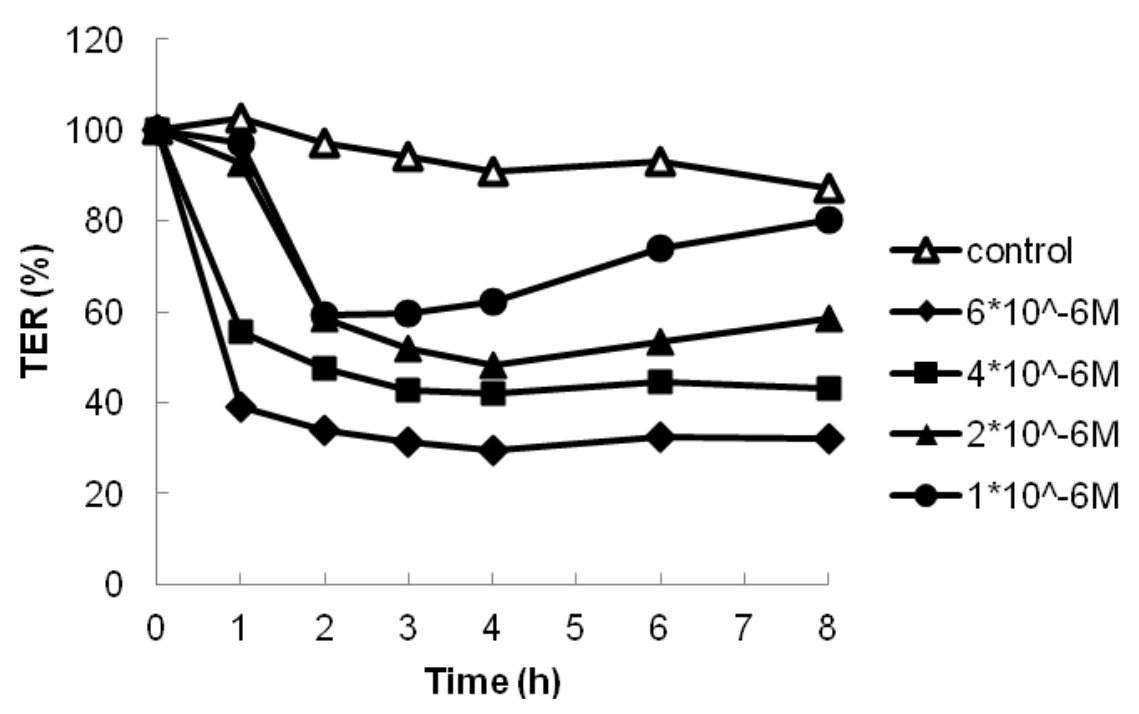

Fig. 1A

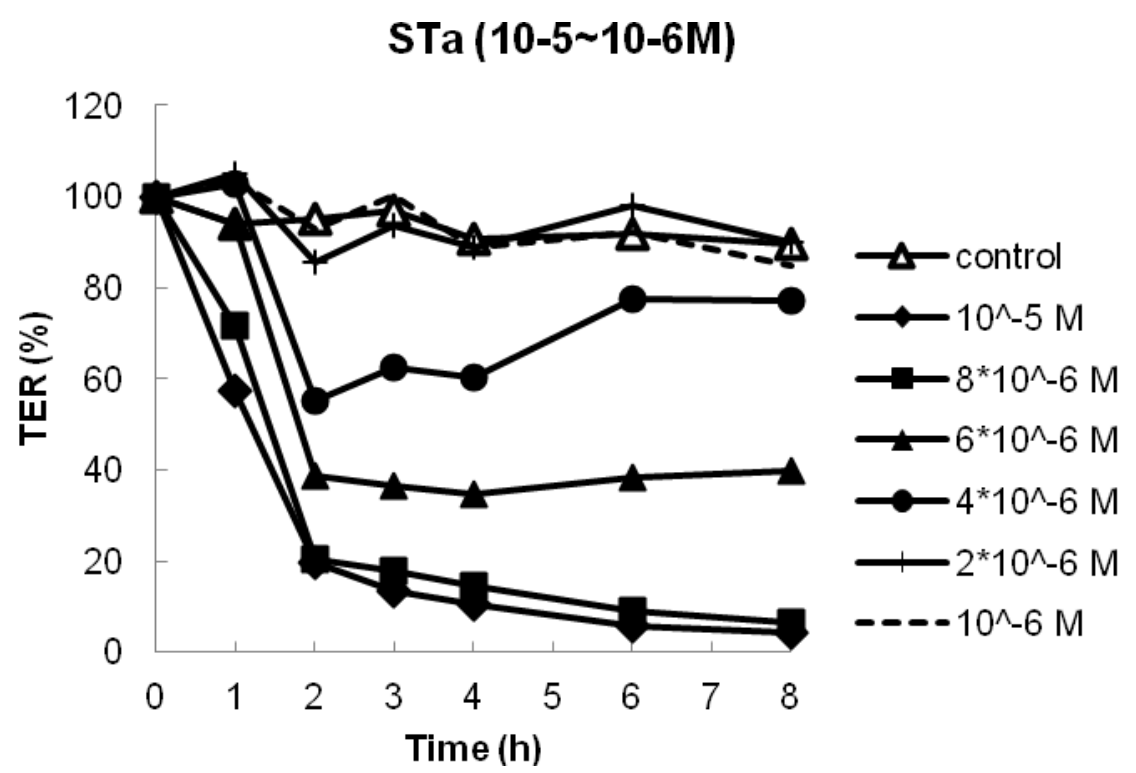

Fig. 1B 


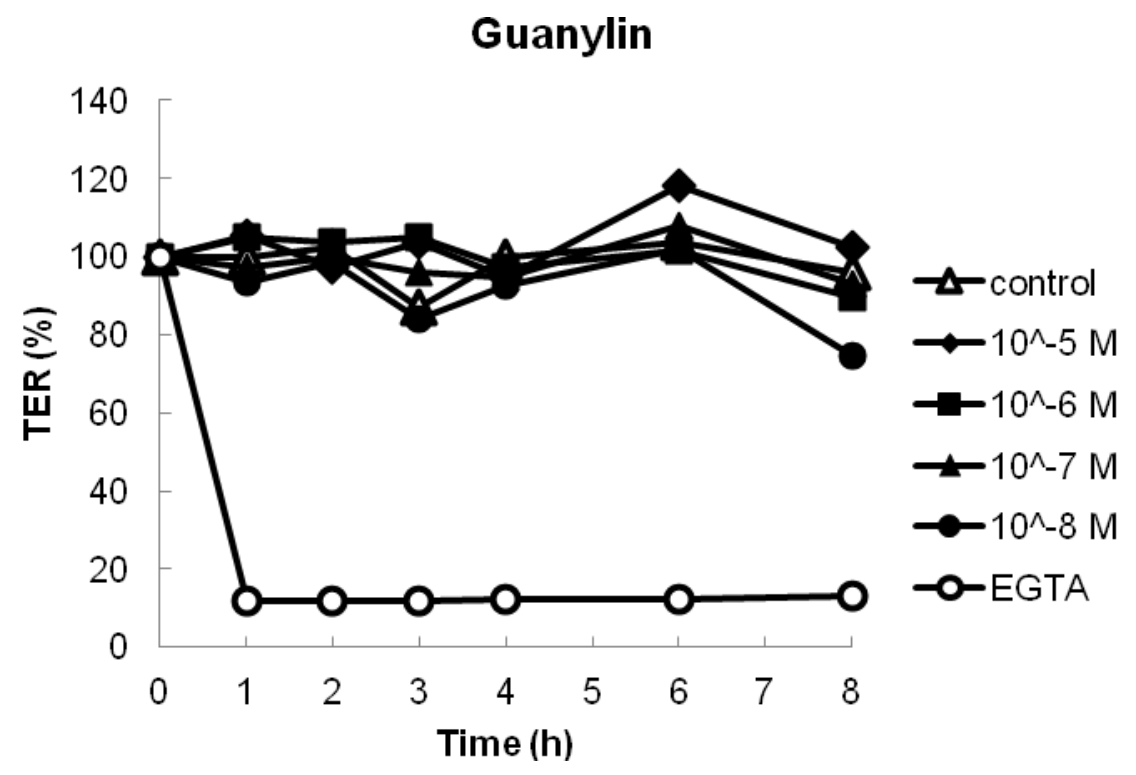

Fig. 1C 


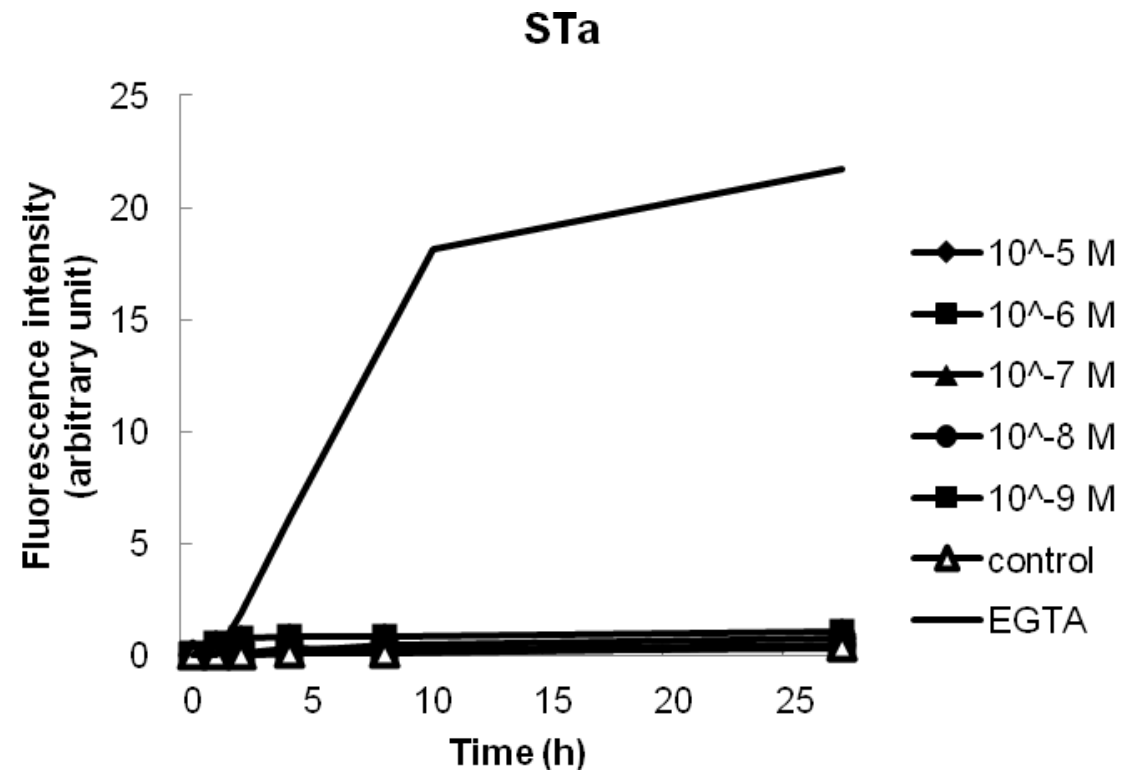

Fig. 2A

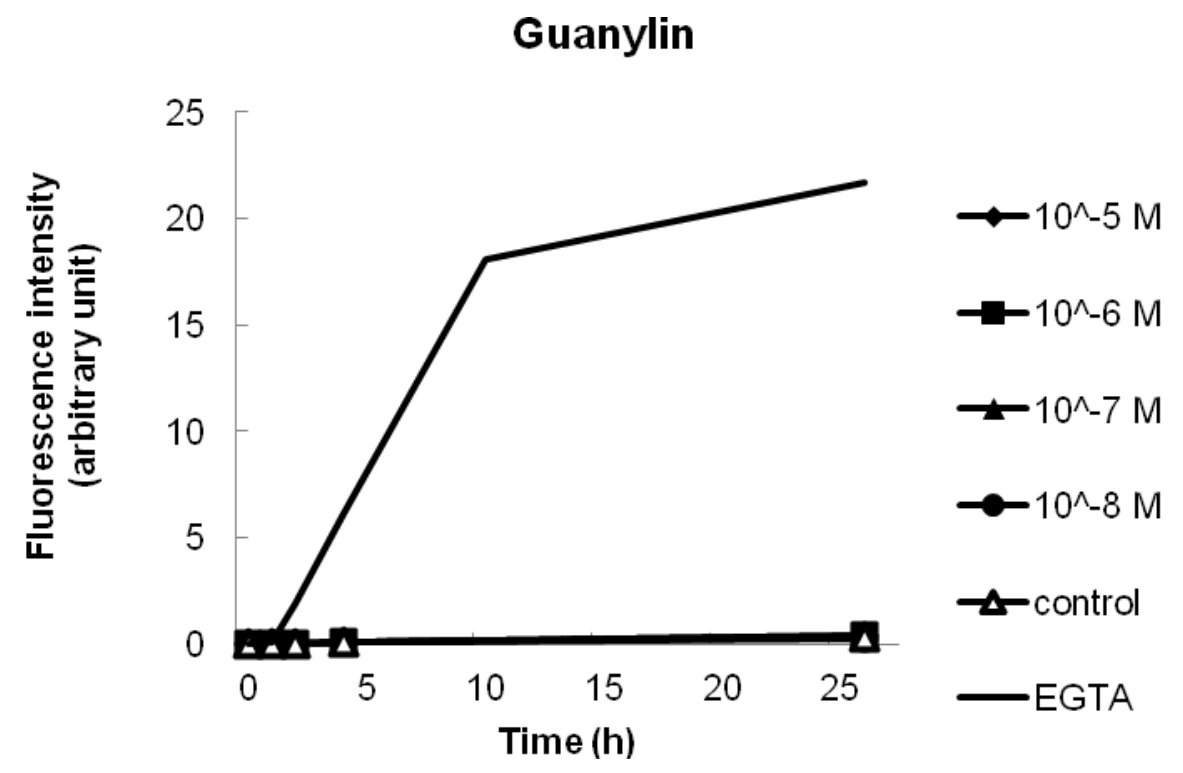

Fig. 2B 
Forskolin

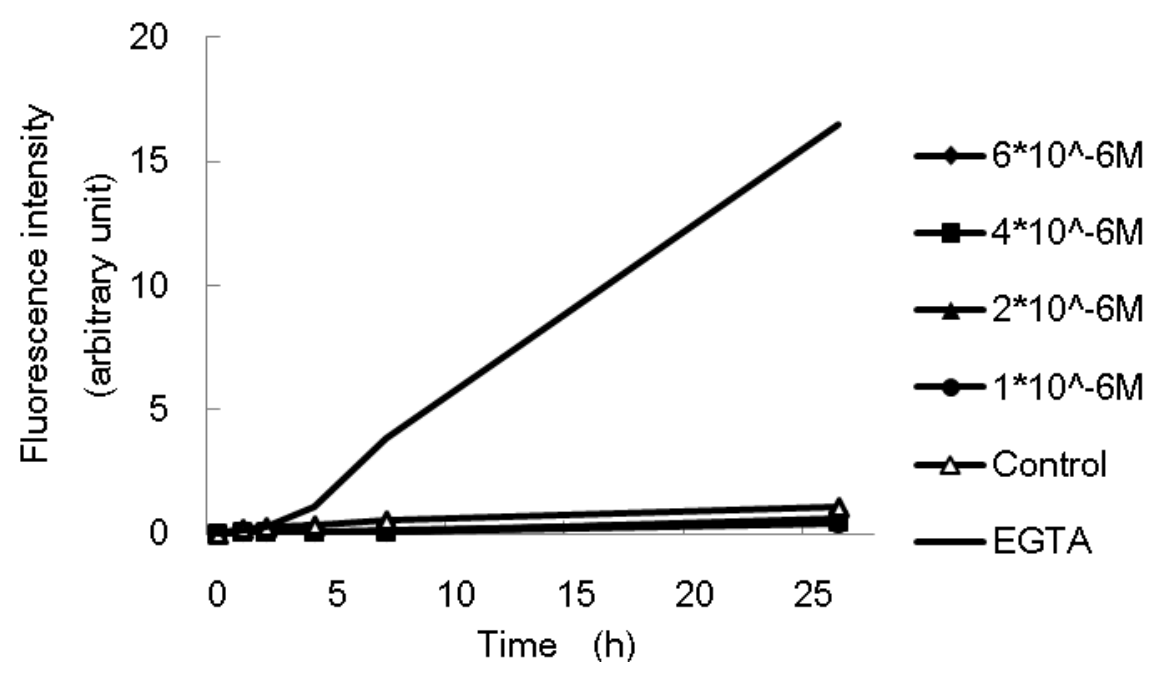

Fig. 2C 